\title{
TORSION-FREE ABELIAN GROUPS WITH PRESCRIBED FINITELY TOPOLOGIZED ENDOMORPHISM RINGS
}

\author{
MANFRED DUGAS AND RUDIGER GÖBEL
}

\begin{abstract}
We will show that any complete Hausdorff ring $R$ which admits, as a basis of neighborhoods of 0 , a family of right ideals $I$ with $R / I$ cotorsion-free can be realized as a topological endomorphism ring of some torsion-free abelian group with the finite topology. This theorem answers a question of A. L. S. Corner (1967) and can be used to provide examples in order to solve a problem (No. 72) in L. Fuchs' book on abelian groups.
\end{abstract}

Introduction. If $G$ is an abelian group, let $\operatorname{End}(G)$ be the endomorphism ring of $G$. Then $\operatorname{End}(G)$ can be given the finite topology, which is also used in functional analysis: If $E \subseteq G$ is a finite subset, let $U_{E}=\{\sigma \in \operatorname{End}(G), E \sigma=0\}$. Then $U_{E}$ is a right ideal of $\operatorname{End}(G)$, and the set of all $U_{E}$ where $E$ ranges over all finite subsets of $G$ constitutes a subbasis of neighborhoods of 0 in $\operatorname{End}(G)$. This topology is evidently Hausdorff and $\operatorname{End}(G)$ is a complete ring (cf. L. Fuchs [9, Vol. II, p. 221, Theorem 107.1]). The finite topolgoy is particularly important in the case of torsion groups (cf. e.g. W. Liebert [15]). It also plays an eminent role for torsion-free groups. The main interest in the torsion-free case lies in its application to decomposition theorems with infinitely many summands, since infinite sums can be treated only with the help of topology. In order to prove the existence of such groups with properties depending on their topological endomorphism rings, we ask the converse problem: Given a ring $R$ with a complete Hausdorff topology generated by a subbase of 0 of right ideals, find a group $G$ such that $R \cong \operatorname{End}(G)$ as topological rings.

In $[2$, p. 60 , Theorem 1.1], A. L. S. Corner characterized all rings $R$ which are endomorphism rings of some countable reduced torsion-free abelian group. This is the case iff $R$ is a complete Hausdorff topological ring which admits as a basis of neighborhoods of 0 a descending sequence of right ideals $N_{\kappa} \triangleleft R$ for $\kappa \in \omega$ such that $R / N_{\kappa}$ is countable, reduced and torsion-free. In the same paper Corner also announces a generalization to the uncountable case: If $R$ satisfies some residually countability condition, then a similar topological realization theorem can be derived (cf. A. L. S. Corner [2, p. 63 ff.]).

This very interesting result, which depends on a theorem in Linear Algebra, has not yet been published. Therefore, several authors have published approaches towards and some deviations to Corner's theorem, as for example G. D'Este $[4,5]$

Received by the editors December 7, 1982.

1980 Mathematics Subject Classification. Primary 20K20, 20K30.

Key words and phrases. Torsion-free abelian groups, topological endomorphism ring.

(c)1984 American Mathematical Society $0002-9939 / 84 \$ 1.00+\$ .25$ per page 
and $\mathrm{A}$. Orsatti $[\mathbf{1 7}, \mathbf{1 8}]$. In this paper we will prove a very general realization theorem for topological rings, which obviously contains Corner's theorem and other results, and leads to further representations. The fact that $\mathbf{Q} \oplus \mathbf{Q}, I_{p} \oplus I_{p}$ and $Z_{p} \oplus Z_{p}$ are never endomorphism rings forces us to exclude $\mathbf{Q}$ (= rational numbers), $I_{p}$ (= $p$-adic integers) and $Z_{p}(Z / p Z)$. Using the terminology from $[10,11]$ we will say that an abelian group $G$ is cotorsion-free if and only if $\mathbf{Q} \nsubseteq G, I_{p} \nsubseteq G$ and $Z_{p} \not G$ for all primes $p$. This is equivalent to saying that $G$ contains no cotorsion subgroups except 0 . Further characterizations of cotorsion-free groups are derived in $[6,7,8,10,11,13]$. We will prove the following

THEOREM. The following conditions on a ring $R$ are equivalent:

(1) $R$ is a complete Hausdorff topological ring which admits as a basis of neighborhoods of 0 a family of right ideals $I$ such that $R / I$ is cotorsion-free.

(2) There is a cotorsion-free abelian group $G$ such that $R \cong \operatorname{End}(G)$ as topological rings.

In fact, we can find a semirigid class which is not a set of groups $G$ with property (2). If $G$ and $H$ are in this class and $|G|>|H|$, then $\operatorname{Hom}(G, H)=0$. The existence of a semirigid class can be derived combining this Theorem and methods from $\mathrm{R}$. Göbel and S. Shelah [12]. The Theorem can also be generalized to modules over Dedekind domains $A$ if and only if $A$ is neither a field nor a complete discrete valuation ring. In this case $R$ is an $A$-algebra and $R / I$ and $G$ are cotorsion-free $A$-modules. Then the Theorem holds verbatim. This can be seen by combining the methods and results of this paper and those of M. Dugas and R. Göbel [8]. However, in order to clarify the basic points of the construction we will restrict ourselves to abelian groups.

The Theorem answers a question in A. L. S. Corner [2, p. 63]. It can also be used to derive a class of counterexamples to Corner's problem stated as Problem 72, p. 183 in Volume II of L. Fuchs [9]:

Problem. Does there exist a group such that every summand $\neq 0$ is a direct sum of infinitely many subgroups $\neq 0$ ?

We will derive even stronger examples: If $\boldsymbol{\aleph}$ is any cardinal, there is a cotorsion-free group $G=G(\boldsymbol{\aleph})$ such that every summand $\neq 0$ decomposes into at least $\aleph$ summands $\neq 0$, cf. $\S 4$. For this application we will use a topological ring constructed by A. L. S. Corner [3]. We also would like to point out that Corner himself gave an example in a letter to L. Fuchs some years ago.

2. Set theoretical preliminaries. We will use some elementary set theoretical notations and ideas from [8]. For the convenience of the reader we will summarize these definitions and results. For detailed proofs we refer to $[7,8]$.

Let $X \backslash Y=\{x \in X, x \notin Y\}$ and $|X|=$ cardinality of $X$. In particular, let $\omega=\aleph_{0}$ $=$ first infinite cardinal. An ordinal $\alpha$ will be identified with the set of all ordinals less than $\alpha$, and a cardinal $\kappa$ will be identified with the ordinal

$$
\inf \{\beta, \beta \text { ordinal, }|\beta|=\kappa\} \text {. }
$$


The set $C \subseteq \alpha$ is cofinal in $\alpha$ if $\sup C=\alpha$ and $\operatorname{cf}(\alpha)$ will denote the ordinal $\inf \{|X|, X$ is cofinal in $\alpha\}$. The cardinal $\kappa$ is regular if $\kappa=\operatorname{cf}(\kappa)$ and singular if $\operatorname{cf}(\kappa)<\kappa$. The cardinal $\kappa$ is a strong limit cardinal if $2^{\lambda}<\kappa$ for every cardinal $\lambda<\kappa$.

If $\left\{X_{i}, i \in I\right\}$ is a family of disjoint sets, then $\prod_{i \in I} X_{i}$ is the cartesian product. If $f$ : $X \rightarrow Y$ is a map and $U \subseteq X$, then $\left.f\right|_{U}$ denotes the restriction of $f$ to the set $U$.

(2.1) If $\kappa$ is a strong limit cardinal, then $2^{\kappa}=\kappa^{\mathrm{cf}(\kappa)}$.

(2.2) If $\rho$ is a cardinal, there is a strong limit cardinal $\lambda$ such that $\operatorname{cf}(\lambda)>\rho$.

(2.3) Let $F, T$ be collections of countable subsets of $\kappa$ such that the following hold:

(a) If $f, g \in F$ and $|f \cap g|=\boldsymbol{\aleph}_{0}$, then $f=g$.

(b) $|T|<|F|$.

(c) $2^{\aleph_{0}}<|F|$.

Then there is a subset $F^{\prime} \subseteq F$ such that $|F|=\left|F^{\prime}\right|$ and $f \in F^{\prime}, t \in T$, with $|t \cap f|=\boldsymbol{\aleph}_{0}$ implying $t=f$.

(2.4) “CiA-LEMmA". Let $\left\{X_{\alpha}, \alpha<\kappa\right\}$ be a family of disjoint sets such that $\left|X_{\alpha}\right|^{|\alpha|}=$ $\left|X_{\alpha}\right| \leqslant\left|X_{\beta}\right|$, for all $\alpha \leqslant \beta<\kappa$. Then there is $F \subseteq \prod_{\alpha \in \kappa} X_{\alpha}$ with $|F|=\left|\prod_{\alpha \in \kappa} X_{\alpha}\right|$ and

(*) If $f, g \in F$ and $\sup \{\nu \in \kappa, f(\nu)=g(\nu)\}=\kappa$, then $f=g$.

(2.5) Let $\mu$ be a strong limit cardinal with $\boldsymbol{\kappa}_{0}<\operatorname{cf}(\mu)$. Let $F$ be a collection of countable subsets of $\mu$ such that $|F|=\lambda^{*}$ for some cardinal $\lambda^{*} \geqslant \operatorname{cf}(\mu)$. Then there is a subset $F^{\prime} \subseteq F$ and a cardinal $\rho<\mu$ such that $\sup f \leqslant \rho$ for all $f \in F^{\prime}$ and $|F|=\left|F^{\prime}\right|$.

Definition 2.6. Let $\mu$ be a singular strong limit cardinal and $\stackrel{\rho}{\mu}=\{\lambda, \lambda$ be a strong limit cardinal, $\operatorname{cf}(\mu)<\lambda<\mu, \operatorname{cf}(\lambda)=\omega\}$. A subset $X \subseteq \lambda \in \stackrel{\rho}{ }$ is $\lambda$-big, if there is a strictly increasing sequence $\lambda_{n} \in \stackrel{\circ}{\mu}$ with $\sup _{n \in \omega} \lambda_{n}=\lambda$ and $\left|X \cap \lambda_{n}^{+}\right|=\lambda_{n}^{+}$ for all $n \in \omega$.

(2.7) If $X \subseteq \lambda \in \stackrel{\circ}{\mu}$ is $\lambda$-big, then $\left|\Pi_{n \in \omega}\left(X \cap \lambda_{n}^{+}\right) \backslash \lambda_{n-1}^{+}\right|=2^{\lambda}$.

(2.8) Let $\kappa$ be an infinite cardinal and $\mu$ a singular strong limit cardinal with $\kappa<\operatorname{cf}(\mu)$. If $X \subseteq \mu$, let $C_{X}$ be all subsets of $X \times \kappa$ of cardinality $\leqslant \aleph$. If $\varphi: C_{\mu} \rightarrow C_{\mu}$ is a map and $\lambda_{0}<\mu, Y \subseteq \mu$ with $|Y|<\operatorname{cf}(\mu)$, there is a $\lambda \in \stackrel{\circ}{\mu}$ with $\lambda_{0}<\lambda$ and a $\lambda$-big set $X$ such that $\varphi\left(C_{X}\right) \subseteq C_{\lambda}$ and $Y \subseteq X$.

3. Proof of the Theorem. (2) $\rightarrow(1)$. Since $R \cong \operatorname{End}(G)$ as a topological ring, by our remarks in $\S 1, R$ is a complete Hausdorff topological ring. If $E$ is a finite subset of $G$, then $\operatorname{End}(G) / U_{E}$ is isomorphic with a subgroup of the direct sum of $|E|$ copies of $G$, because $U_{E}$ is the kernel of the homomorphism $\operatorname{End}(G) \rightarrow G^{E}\left(\sigma \rightarrow(e \sigma)_{e \in E}\right)$. Since $G$ is cotorsion-free, $\operatorname{End}(G) / U_{E}$ is also cotorsion-free. Using the topological isomorphism $R \cong \operatorname{End}(G)$, we see that $R$ admits as a basis of neighborhoods of 0 a family of right ideals $I$ such that $R / I$ is cotorsion-free.

$(1) \rightarrow(2)$. Let $g$ be a set of right ideals of $R$ which constitutes a subbasis of neighborhoods of 0 of a complete Hausdorff topology of $R$ such that $R / I$ is cotorsion-free for all $I \in \mathcal{g}$. We choose a strong limit cardinal $\mu$ with $|R|^{\boldsymbol{N}_{0}} \cdot|q|<$ $\operatorname{cf}(\mu)<\mu$, and let $B_{I}=\bigoplus_{\alpha<\mu}(I, \alpha) R$ with generators $(I, \alpha) \operatorname{such}$ that $\operatorname{ann}_{R}(I, \alpha)=I$ for all $I \in \mathcal{G}$ and $B=\bigoplus_{I \in \mathcal{G}} B_{I}$. If $X$ is any torsion-free reduced abelian group, define $\hat{X}$ to be the completion of $X$ in the Z-adic topology. Hence $x \in \hat{B}$ can be written as $x=\Sigma_{n \in \omega}\left(I_{n}, \alpha_{n}\right) r_{n}$ such that $\left\{n, r_{n}+I_{n} \notin m !\left(R / I_{n}\right)^{\wedge}\right\}$ is finite for all $m \in \omega$. Let 
$[x]=\left\{\left(I_{n}, \alpha_{n}\right),\left(I_{n}, \alpha_{n}\right) \cdot r_{n} \neq 0\right\}$ be the support of $x, \llbracket x \rrbracket=\{\alpha \in \mu$, there is $I \in f$ such that $(I, \alpha) \in[x]\}$ and the norm $\|x\|=\sup \llbracket x \rrbracket$. If $X \subseteq \mu$, we also use $\|X\|=$ $\sup (X)$. If $\lambda \in \mu$, define $\hat{B}_{\lambda}=\{x \in \hat{B},\|x\| \leqslant \lambda\}$ and let $\stackrel{\circ}{\mu}=\{\lambda<\mu, \lambda$ be a strong limit cardinal, $\operatorname{cf}(\mu)<\lambda, \operatorname{cf}(\lambda)=\omega\}$ and $\gamma=\min (\stackrel{\circ}{\mu}) \in \stackrel{\circ}{\mu}$ (compare $\S 2$ ). Then $\stackrel{\mu}{\mu}$ is a stationary subset of $\mu$. The basic construction is similar to [8]. Hence, we will adapt some terminology.

If $X \subseteq \mu$, let $B_{X}=\bigoplus_{I \in \mathcal{I}} \bigoplus_{\alpha \in X}(I, \alpha) R$, and consider all pairs $\left(\varphi, B_{X}\right)$ such that

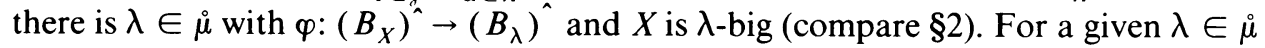
there are (at most) $2^{\lambda}$ such pairs. Hence we can label these pairs as $\left(\varphi_{\alpha}^{\lambda}, P_{\alpha}^{\lambda}\right)$ with $\alpha \in 2^{\lambda}$. Let $L=\left\{(\lambda, \alpha), \lambda \in \stackrel{\mu}{, \alpha} \in 2^{\lambda}\right\}$ be the list of all parameters given with the lexicographical order. Also choose an enumeration of $g \times g=\left\{\left(I_{\alpha}, J_{\alpha}\right), \alpha \in 2^{\gamma}\right\}$. Obviously, we will allow repetitions. Next we define the generators $O_{\alpha}^{\lambda}$ of $G$. There exists a system $\left\{\left\{\gamma_{n}^{\alpha} \in \mu, n \in \omega\right\}, \alpha \in 2^{\gamma}\right\}$ such that $\sup _{n \in \omega} \gamma_{n}^{\alpha}=\gamma$ for all $\alpha \in 2^{\gamma}$ and $\left\{\gamma_{n}^{\alpha} \in \mu, n \in \omega\right\} \cap\left\{\gamma_{n}^{\beta}, n \in \omega\right\}$ is finite for all $\alpha \neq \beta$. This follows from our CIA-Lemma 2.4 .

If $\lambda=\gamma$, we set

$$
O_{\alpha}^{\gamma}=\sum_{n \in \omega}\left(I_{\alpha}, \gamma_{2 n}^{\alpha}\right)(2 n) !+\sum_{n \in \omega}\left(J_{\alpha}, \gamma_{2 n+1}^{\alpha}\right)(2 n+1) !
$$

for all $\alpha \in 2^{\gamma}$. The other elements $O_{\alpha}^{\lambda}, b_{\alpha}^{\lambda}$ and a subset $C \subseteq L$ are defined by transfinite induction on $L$ subject to the following conditions:

Assume that all elements $O_{\beta}^{\kappa}, b_{\beta}^{\kappa}$ for $(\kappa, \beta)<(\lambda, \alpha)$ and $(\kappa, \beta) \in L$ are already constructed. Then we define $G_{\alpha}^{\lambda}=\left\langle B, O_{\beta}^{\kappa} R,(\kappa, \beta) \in L,(\kappa, \beta)<(\lambda, \alpha)\right\rangle^{*} \subseteq \hat{B}$ first. Recall that $U^{*} \subseteq G$ denotes the purification of $U$ in $G$. Since the groups are torsion-free, $U^{*}$ is generated by $U$ and all preimages of the torsion elements in $G / U$. Then we require

(a) $O_{\alpha}^{\lambda} \in P_{\alpha}^{\lambda}$ and $b_{\alpha}^{\lambda} \in\left(B_{\lambda}\right)^{\wedge}$,

(b) $O_{\alpha}^{\lambda}=w_{\alpha}^{\lambda}+\sum_{n \in \Delta}\left(I, \lambda_{n}\right) n$ ! such that $I \in \mathscr{G}, \operatorname{ann}_{R}\left(w_{\alpha}^{\lambda}\right) \supseteq J$ for some $J \in \mathscr{q}$; $\left\{\lambda_{n}, n \in \omega\right\}$ is a strictly monotone sequence converging to $\lambda,\left\|w_{\alpha}^{\lambda}\right\|<\lambda$ and $\Delta \subseteq \omega$ is infinite,

(c) $\left\|\llbracket O_{\alpha}^{\lambda} \rrbracket \cap\left(\llbracket O_{\beta}^{\kappa} \rrbracket \cup \llbracket b_{\beta}^{\kappa} \rrbracket\right)\right\|<\lambda$, for all $(\kappa, \beta)<(\lambda, \alpha)$.

If there exist $O_{\alpha}^{\lambda} \in P_{\alpha}^{\lambda}$ such that $b_{\alpha}^{\lambda}=\varphi_{\alpha}^{\lambda}\left(O_{\alpha}^{\lambda}\right)$, and $O_{\alpha}^{\lambda}$ have the properties (a), (b), (c) and $b_{\alpha}^{\lambda} \notin G_{\alpha+1}^{\lambda}$, we choose this pair. Recall, that $\alpha+1$ is the successor ordinal of $\alpha$. In this case we let $(\lambda, \alpha) \in C$. In the opposite case let $(\lambda, \alpha) \notin C$ and choose any elements $O_{\alpha}^{\lambda}$ and $b_{\alpha}^{\lambda}$ with the properties (a), (b), (c). The existence of these elements follows from our set theoretic results (2.3), (2.4), (2.6) and (2.7). A detailed proof is given in [8]. The required group will be $G=\left\langle B, O_{\alpha}^{\lambda} R,(\lambda, \alpha) \in L\right\rangle^{*} \subseteq \hat{B}$. We will reserve the letter $G$ for this particular torsion-free abelian group. First we will show that

(d) $G$ is cotorsion-free.

A group $G$ is cotorsion-free if and only if $\operatorname{Hom}(\hat{\mathbf{Z}}, G)=0$. Since $\mathbf{Z}$ is an $h$-local ring, this is equivalent to $\operatorname{Hom}\left(J_{p}, G\right)=0$ for all primes $p$. Therefore, let $\varphi \in$ $\operatorname{Hom}\left(J_{p}, G\right)$ and 1 be the unit element of $J_{p}$. Hence $\varphi(1) \in G$ and since $G=$ $\left\langle B, O_{\alpha}^{\lambda} R,(\lambda, \alpha) \in L\right\rangle^{*}$ is torsion-free, we may assume that $\varphi(1)=O_{\alpha}^{\lambda} \cdot r+c+b$, such that $\|c\|<\lambda$ and $b \in B$, where $c$ is a linear combination of certain elements $O_{\beta}^{\kappa}$ 
and $r \in R / I$. Assume first, that $O_{\alpha}^{\lambda} r \neq 0$. Since $J_{p}$ is divisible by all primes different from $p, \varphi\left(J_{p}\right)$ also has this property. However, $G \subseteq \hat{B}$ is reduced. Hence, $r \in$ $R / I \backslash p^{\omega}(R / I)$ implies $r \pi \neq 0$ for all $\pi \in J_{p} \backslash 0$, and we conclude $O_{\alpha}^{\lambda} r \pi \neq 0$ (compare [6, p. 324, Lemma 2.5]). Since $\varphi(\pi) \in G$, we can find $n_{\pi} \in \mathbf{N}$ and $t_{\pi} \in R / I$ such that $\varphi(\pi) \cdot n_{\pi}=\varphi(1) \pi \cdot n_{\pi}=O_{\alpha}^{\lambda} r \pi n_{\pi}+c \pi n_{\pi}+b \pi n_{\pi} \equiv O_{\alpha}^{\lambda} t_{\pi}+d \bmod B$ where $\|d\|<\lambda$.

The definition of $O_{\alpha}^{\lambda}$ and a support argument implies $n ! \cdot r \cdot \pi n_{\pi}=n ! \cdot t_{\pi}$, for almost all $n \in \mathbf{N}$. Since $R / I$ is torsion-free, we derive $r \cdot \pi \cdot n_{\pi}=t_{\pi} \in R / I \cap$ $n_{\pi}(R / I)^{\wedge}$. However, $R / I$ is pure in $(R / I)^{\wedge}$ and, therefore, $r \cdot \pi \cdot n_{\pi}=s_{\pi} \cdot n_{\pi}$ for some $s_{\pi} \in R / I$. We conclude that $r \cdot \pi \in R / I$ and $\psi: J_{p} \rightarrow R / I(\pi \rightarrow r \cdot \pi)$ is a homomorphism. Since $R / I$ is cotorsion-free, this homomorphism must vanish and, in particular, $\psi(1)=r=0$. Induction shows that $\varphi(1) \in B$. Therefore, $\varphi(1) \in$ $\bigoplus_{i=1}^{n}\left(I_{i}, \alpha_{i}\right) R / I=D$ and $\varphi\left(J_{p}\right) \subseteq G \cap \hat{D}$.

If $(I, \alpha) r \in G \cap \hat{D}$ and $r \in(R / I) \hat{)}$, then $(I, \alpha) \cdot r n=(I, \alpha) t$ for some $n \in \mathbf{N}$ and $t \in R / I$. Hence $r n=t$ and the same purity-argument shows $r \in R / I$. We conclude that $G \cap \hat{D}=G \cap D \subseteq D$, which is cotorsion-free. Since $\varphi\left(J_{p}\right) \subseteq D$, $\varphi\left(J_{p}\right)$ is also cotorsion-free and $\varphi\left(J_{p}\right)$ is cotorsion. This is only possible if $\varphi=0$.

Since $R$ acts by multiplication on $G$ and $B \subseteq G$, we derive $R \subseteq$ End $G$. In addition, we will need

(e) $R$ is a pure subgroup of $\operatorname{End}(\hat{B})$.

Let $\varphi \in \operatorname{End}(\hat{B}), n$ a natural number and $r \in R$ such that $n \varphi=r$. In particular, $(I, \alpha) r=(I, \alpha) \varphi n$ for the generators $(I, \alpha)$ of $B$. Since $R / I$ is torsion-free, we can find $x_{I} \in R$ with $(I, \alpha) \varphi=(I, \alpha) x_{I}$. We conclude that $r-n x_{I} \in I$.

If $J \subseteq I \in \mathcal{G}$, then $r-n x_{J} \in J \subseteq I$ and $n x_{I}-n x_{J} \in I$. Since $n\left(x_{I}-x_{J}\right) \in I$ and $R / I$ is torsion-free, we derive $x_{I}-x_{J} \in I$ for all $J \subseteq I$. This means that $\left(x_{I}\right)_{I \in g}$ is a Cauchy net. Since $R$ is complete by (1), we can find $x \in R$ such that $x-x_{I} \in I$ for all $I \in g$. In particular, $n x-n x_{I} \in I$ and $r-n x_{I} \in I$ from above. Therefore, $r-n x \in I$ for all $I \in \mathcal{G}$. The topology on $R$ is a Hausdorff topology and hence $\cap \mathcal{g}=0$. We conclude that $r=n x$ and therefore $n x=n \varphi$. Because $\operatorname{End}(\hat{B})$ is torsion-free, $\varphi=x \in R$ also.

Since $B=\bigoplus_{I \in \mathcal{G}} B_{I}$, the elements in $R$ act by multiplication on $B_{I}$ and this action can be extended such that the elements of $\prod_{I \in \mathcal{g}} R$ act canonically as endomorphisms on $\hat{B}$. Hence, $\Pi_{I \in \mathcal{g}} R \subseteq \operatorname{End}(\hat{B})$.

(f) If $\varphi \in \operatorname{End}(\hat{B}) \backslash \prod_{I \in \mathcal{G}} R$, then there exist $a \in\left(B_{I}\right)^{\wedge}$ for some $I \in \mathcal{g}$, such that $n \varphi(a) \notin G+a R$ for all $n \in \mathbf{N}$.

First, we will show by the way of contradiction that we can find $I \in \mathcal{G}$ such that $n \varphi-\left.r\right|_{\left(B_{1}\right)} \neq 0$ for all $r \in R$ and $n \in \mathbf{N}$. If this is not true, we can choose $r_{I} \in R$ and $n \in \mathbf{N}$ such that $\left.\left(n_{I} \varphi-r_{I}\right)\right|_{\left(B_{I}\right)^{\wedge}}=0$ for all $I \in \mathcal{G}$. Therefore, $(I, \alpha) \varphi \cdot n_{I}=$ $(I, \alpha) \cdot \dot{r_{I}}$ which implies $r_{I}+I=n_{I} s_{I}+I$, for some $s_{I} \in R$. Therefore, $n_{I} \varphi-$ $\left.n_{I} s_{I}\right|_{\left(B_{I}\right)^{\wedge}}=0$ and, equivalently, $\left.n_{I}\left(\varphi-s_{I}\right)\right|_{\left(B_{I}\right)^{\wedge}}=0$. Because $\left(B_{I}\right)^{\wedge}$ is torsion-free, $\varphi-\left.s_{I}\right|_{\left(B_{I}\right)^{\wedge}}=0$ is also. Therefore, $\varphi=\left(s_{I}\right)_{I \in J} \in \prod_{I \in J} R$, which is a contradiction. Hence, our first claim holds.

Now we can choose $I \in \mathcal{g}$ such that $(n \varphi-r)\left(B_{I}\right)^{\wedge} \neq 0$ for all $n \in \mathbf{N}$ and $r \in R$. The group $\left(B_{I}\right)^{\wedge}$ is cotorsion by construction. Therefore, $(n \varphi-r)\left(B_{I}\right)^{\wedge}$ is also a nontrivial cotorsion group (cf. L. Fuchs $[9$, p. 163, Theorem 39.1; p. 234, 
Proposition 54.1]). Since $G$ is cotorsion-free by (d), we conclude $(n \varphi-r)\left(B_{I}\right)^{\wedge} \nsubseteq G$. Therefore, we can find elements.

$\left(1^{*}\right) b_{r n} \in\left(B_{I}\right)^{\wedge}$ such that $(n \varphi-r)\left(b_{r n}\right) \notin G$ for all $n \in \mathbf{N}, r \in R$.

Since $|R|^{\aleph_{0}} \cdot|J|<\operatorname{cf}(\mu)$, we can choose $\lambda \in \stackrel{\mu}{\mu}$ such that $\left\|b_{r n}\right\|<\lambda$ and $\left\|\varphi\left(b_{r n}\right)\right\|<\lambda$ for all $r \in R$ and $n \in \mathbf{N}$. Pick any $O_{\alpha}^{\lambda}$ and choose a strictly increasing sequence $\lambda_{n} \in\left[O_{\alpha}^{\lambda}\right]$ such that $\sup _{n \in \omega} \lambda_{n}=\lambda$ and consider $a^{0}=\Sigma_{n \in \omega}\left(I, \lambda_{2 n}\right)(2 n)$ !. An easy support argument similar to [8] shows that $a^{0}$ has the property

$\left(2^{*}\right)\left[a^{0}\right] \backslash F \not[g]$ for all finite subsets $F$ of $\left[a^{0}\right]$ and all $g \in G$.

If $a=a^{0}$ satisfies (f), the proof is finished. Therefore, let $(n \varphi-r)\left(a^{0}\right) \in G$ for some $n \in \mathbf{N}$ and $r \in R$. Hence, we can pick $b_{r n}$ from $\left(1^{*}\right)$ and consider $a^{*}=a^{0}+b_{r n}$. Again, we can assume $(m \varphi-s)\left(a^{*}\right) \in G$ for some $m \in \mathbf{N}$ and $s \in R$. Subtraction of $(n m \varphi-s n)\left(a^{*}\right) \in G$ from $(n m \varphi-r m)\left(a^{0}\right) \in G$ leads to $n m \varphi\left(b_{r n}\right)-s n b_{r n}+$ $a^{0}(r m-s n) \in G$. Using the support argument and $\left(2^{*}\right)$, we derive $a^{0}(r m-s n)=0$. Since $\operatorname{ann}_{R}\left(a^{0}\right)=I$, we conclude $r m-s n \in I$. However, $b_{r n} \in\left(B_{I}\right)^{\wedge}$ and therefore we may exchange $r m$ and $s n$ to derive $n m \varphi\left(b_{r n}\right)-r m b_{r n} \in G$ from the last inclusion. Since $\hat{B} / G$ is torsion-free and $m\left(n \varphi\left(b_{r n}\right)-r b_{r n}\right) \in G$ we obtain a contradiction $n \varphi\left(b_{r n}\right)-r b_{r n} \in G$ with (1*). The claim (f) can be used to derive

(g) $\operatorname{End}(G) \subseteq \prod_{I \in J} R$.

We want to show that an endomorphism $\varphi \in$ End $G \backslash \prod_{I \in J} R$ cannot leave $G$ invariant. From (f) we obtain $a \in\left(B_{I}\right)^{\wedge}$ for some $I \in \mathcal{g}$ such that

$\left(3^{*}\right) n \varphi(a) \notin G+a R$ for all $n \in \mathbf{N}$.

Recall that $B \subseteq G, B$ is dense and $G$ is pure in $\hat{B}$. Hence $\varphi$ has a unique extension to an endomorphism of $\hat{B}$. We will identify these two maps which are used in $\left(3^{*}\right)$.

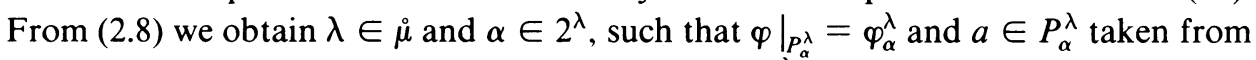
the list $L$. We want to show that $(\lambda, \alpha) \in C$. Since $P_{\alpha}^{\lambda^{\alpha}}=B_{X}$ for some $\lambda$-big set $X$, we can choose $c=\sum_{n \in \omega}\left(I, \lambda_{n}\right) n ! \in P_{\alpha}$, such that $\llbracket c \rrbracket \cap\left(\llbracket O_{\beta}^{\kappa} \rrbracket \cup \llbracket b_{\beta}^{\kappa} \rrbracket\right)$ is finite for all $(\kappa, \beta)<(\lambda, \alpha)$ (compare (2.7)). Assume that $(\lambda, \alpha) \in L \backslash C$ for contradiction. Therefore $\varphi(c) \in\left\langle G_{\alpha}^{\lambda}+c R\right\rangle^{*}$ and we can find $n \in \mathbf{N}$ such that $n \varphi(c) \in G_{\alpha}^{\lambda^{\prime}}+c R$. Using the same argument, we can find $m \in \mathbf{N}$ such that $m \varphi(c+a) \in G_{\alpha}^{\lambda}+(c+a) R$. Therefore, $n \varphi(c)-c r \in G_{\alpha}^{\lambda}$ and $m \varphi(c)+m \varphi(a)-c s-a s \in G_{\alpha}^{\lambda}$ for some $s \in R$. We derive $m n \varphi(c)-m c r \in G_{\alpha}^{\lambda}$ and $m n \varphi(c)+n m \varphi(a)-c n s-a n s \in G_{\alpha}^{\lambda}$; hence, $n m \varphi(a)-a n s+c(m r-n s) \in G_{\alpha}^{\lambda}$. By the support argument, we derive $c(m r-n s)$ $=0$ and since $\operatorname{ann}_{R}(c)=I$ we have $m r-n s \in I$ also. Since $a \in\left(B_{I}\right)^{\wedge}$ we can exchange $n s$ and $m r$ and derive $n m \varphi(a)-a m r \in G_{\alpha}^{\lambda}$. However, $B / G_{\alpha}^{\lambda}$ is torsion-free, which implies $n \varphi(a)-a r \in G_{\alpha}^{\lambda}$, i.e. $n \varphi(a) \in G_{\alpha}^{\lambda}+a R$. This contradicts (f) and therefore $(\lambda, \alpha) \in C$. We conclude $b_{\alpha}^{\lambda}=\varphi_{\alpha}^{\lambda}\left(O_{\alpha}^{\lambda}\right)=\varphi\left(O_{\alpha}^{\lambda}\right) \notin G$. Finally, we show

(h) $\operatorname{End}(G)=R$.

Obviously, $R \subseteq \operatorname{End}(G)$. If $\varphi \in \operatorname{End}(G)$, then $\varphi=\left(s_{I}\right)_{I \in g}$ as shown in $(\mathrm{g})$. We now consider the elements $O_{\alpha}^{\gamma}$ with $\gamma=\min (\dot{\mu})$. Since

$$
O_{\alpha}^{\gamma}=\sum_{n \in \omega}\left(I_{\alpha}, \gamma_{2 n}^{\alpha}\right)(2 n) !+\sum_{n \in \omega}\left(J_{\alpha}, \gamma_{2 n+1}^{\alpha}\right)(2 n+1) !
$$

we have

$$
\varphi\left(O_{\alpha}^{\gamma}\right)=\sum_{n \in \omega}\left(I_{\alpha}, \gamma_{2 n}^{\alpha}\right)(2 n !) \cdot s_{I_{\alpha}}+\sum_{n \in \omega}\left(J_{\alpha}, \gamma_{2 n+1}^{\alpha}\right)(2 n+1) ! s_{J_{\alpha}} \in G .
$$


We want to show that $\left(s_{I}\right)_{I \in \mathcal{G}}$ is a Cauchy-net. If $\varphi\left(O_{\alpha}^{\gamma}\right)$ has finite support, then $s_{I_{\alpha}} \in I_{\alpha}$ and $s_{J_{\alpha}} \in J_{\alpha}$. Therefore, $s_{I_{\alpha}}-s_{J_{\alpha}} \in I_{\alpha}$ if $J_{\alpha} \subseteq I_{\alpha}$. If $\varphi\left(O_{\alpha}^{\gamma}\right)$ has infinite support, there are $r_{\alpha} \in R$ and $n_{0}, m_{\alpha} \in \mathbf{N}$ such that

$$
\begin{aligned}
& m_{\alpha}\left(\sum_{n \geqslant n_{0}}\left(I_{\alpha}, \gamma_{2 n}^{\alpha}\right)(2 n) ! s_{I_{\alpha}}+\sum_{n \geqslant n_{0}}\left(J_{\alpha}, \gamma_{2 n+1}^{\alpha}\right)(2 n+1) ! s_{J_{\alpha}}\right) \\
& \quad \equiv \sum_{n \geqslant n_{0}}\left(I_{\alpha}, \gamma_{2 n}^{\alpha}\right)(2 n) ! r_{\alpha}+\sum_{n \geqslant n_{0}}\left(J_{\alpha}, \gamma_{2 n+1}^{\alpha}\right)(2 n+1) ! r_{\alpha} \bmod B
\end{aligned}
$$

Therefore, $m_{\alpha}(2 n) ! s_{I_{\alpha}}-(2 n) ! r_{\alpha} \in I_{\alpha}$ and $m_{\alpha}(2 n+1) ! s_{J_{\alpha}}-(2 n+1) ! r_{\alpha} \in J_{\alpha}$ for infinitely many $n$. Since $R / I_{\alpha}$ is torsion-free, $m_{\alpha} s_{I_{\alpha}}-r_{\alpha} \in I_{\alpha}$ and $m_{\alpha} s_{J_{\alpha}}-r_{\alpha} \in J_{\alpha}$. If $J_{\alpha} \subseteq I_{\alpha}$, we derive $m_{\alpha}\left(s_{I_{\alpha}}-s_{J_{\alpha}}\right) \in I_{\alpha}$ and also $s_{I_{\alpha}}-s_{J_{\alpha}} \in I_{\alpha}$. Therefore, $\left(s_{I}\right)_{I \in J}$ is a Cauchy-net. The ring $R$ is complete by (1). Therefore $s \in R$, such that $s-s_{I} \in I$ for all $I \in \mathcal{G}$ exists. Hence $\varphi=\left(s_{I}\right)_{I \in J}=(s)_{I \in \mathcal{g}} \in R$. Observe that the finite topology on $\operatorname{End}(G)$ automatically coincides with the topology on $R$.

4. Applications. If we choose the discrete topology on $R$, the Theorem has as an immediate consequence

COROLlARY 1. The following conditions on a ring $R$ are equivalent:

(1) $R$ is cotorsion-free.

(2) There is a cotorsion-free abelian group $G$ such that $R \cong$ End $G$.

This corollary was shown in [8].

Recall that a module $G$ is superdecomposable, if every direct summand $\neq 0$ is a proper direct sum. In [8] we used a module-theoretic form of Corollary 1 to show the existence of superdecomposable modules over Dedekind domains different from fields and complete discrete valuation rings. This was an open question for a large class of Dedekind domains. If $\boldsymbol{N}$ is some cardinal, we will say that a module $G$ is $\boldsymbol{\aleph}$-decomposable if every direct summand $\neq 0$ is a direct sum of at least $\boldsymbol{\aleph}$ nontrivial summands. Therefore superdecomposable coincides with 2-decomposable.

A. L. S. Corner [3] constructed a ring $R=R(\boldsymbol{\aleph})$ with the following properties:

(a) $R$ is commutative and has a complete Hausdorff topology which arises from a set $\mathcal{G}$ of ideals such that $(R / I)^{+}$is free for all $I \in \mathcal{G}$.

(b) If $0 \neq d \in R$ is an idempotent, there are orthogonal idempotents $d_{i} \in R$ for $i \in \boldsymbol{N}$ such that $d=\Sigma_{i \in \boldsymbol{N}} d_{i}$ as topological sum.

The ring is a natural but ingenious $\mathbf{Z}$-semigroup ring construction in the boolean algebra of power sets. With the help of our Theorem we derive

COROLlARY 2. If $\aleph$ is a cardinal, then there exists a $\aleph$-decomposable abelian group.

A simple modification of Corner's ring and the application of a module theoretic version of our Theorem (cf. §1) leads to the existence of $\aleph$-decomposable modules over Dedekind domains if and only if the ground ring is neither a field nor a complete discrete valuation ring. Corollary 2 gives a large supply of counterexamples of L. Fuchs [9, Vol. II, p. 183, Problem 72]. 
There is another surprising application of our Theorem. Let $\mathcal{E}=\left\{R_{i}, i \in I\right\}$ be a collection of rings with cotorsion-free additive groups $R_{i}^{+}$. We call a system $\left\{G_{i}, i \in I\right\}$ of abelian groups $\mathcal{E}$-rigid if $\operatorname{End}\left(G_{i}\right) \cong R_{i}$ and $\operatorname{Hom}\left(G_{i}, G_{j}\right)=0$ for all $j \neq i \in I$. This extends a well-known definition, compare L. Fuchs [9, Vol. II, p. 124]. Now we consider our favoured ring $R=\prod_{i \in I} R_{i} e_{i}$ a generalization of the Baer-Specker-ring equipped with the product topology. The product topology is generated by all ideals $\prod_{I \backslash F} R_{i}$ with finite $F$ with the canonical imbedding into $R$. Therefore the assumptions (1) of our Theorem are satisfied and we can find a cotorsion-free group $G$ with End $G \cong R$ as topological rings. Since $1=\sum_{i \in I} e_{i}$ in the product topology, we conclude $G=\bigoplus_{i \in I} G e_{i}$ and $R_{i} \cong R_{i} e_{i}=R e_{i}=\operatorname{End}\left(G e_{i}\right)$. On the other hand, $\operatorname{Hom}\left(G_{i}, G_{j}\right)=0$ for $i \neq j$ as follows from $e_{i} e_{j}=0$. Hence, we derive a very strong representation-theorem as

COROllaRY 3. If $\mathcal{E}=\left\{R_{i}, i \in \boldsymbol{\aleph}\right\}$ is any family of rings $R_{i}$ with cotorsion-free additive group, then there is an $\mathcal{E}$-rigid system $\left\{G_{i}, i \in \boldsymbol{N}\right\}$ of cotorsion-free abelian groups.

Finally, we consider the ring $R=\mathbf{Z}[[x]]$ of all formal power series, which is complete in the topology $\tau$ generated by the ideals $x^{n} R$ for all $n \in \omega$. Since $R / x^{n} R$ has a free additive group, we can apply the Theorem and find $G$ such that $\operatorname{End}(G) \cong R$. The ring $R$ has no idempotents other than 0 and 1 . Hence $G$ is an indecomposable abelian group and, in particular, an indecomposable $R$-module. On the other hand, $G$ is a torsion module. Therefore all well-known splitting properties of torsion modules break down for this module category. Recall that Corollary 1 can be used to build indecomposable torsion-free $R$-modules.

\section{REFERENCES}

1. A. L. S. Corner, Every countable reduced torsion-free ring is an endomorphism ring, Proc. London Math. Soc. (3) 13 (1963), 687-710.

2. Endomorphism rings of torsion-free abelian groups, Proc. Internat. Conf. on the Theory of Groups, Australian National University, Canberra, 1965, Gordon \& Breach, New York, 1967, pp. 59-69.

3. A topological ring with the property that every non-trivial idempotent is the infinite orthogonal sum of idempotents, Abelian Group Theory (Proc. Honolulu, 1983), Lecture Notes in Math., vol. 1006, Springer, Berlin and New York (to appear).

4. G. D'Este, $A$ theorem on the endomorphism ring of reduced torsion-free abelian groups and some applications, Ann. Mat. Pura Appl. (4) 116 (1978), 381-392.

5. _ On topological rings which are endomorphism rings of reduced torsion-free abelian groups, Quart. J. Math. Oxford Ser. (2) 32 (1981), 303-311.

6. M. Dugas and R. Göbel, Every cotorsion-free ring is an endomorphism ring, Proc. London Math. Soc. (3) 45 (1982), 319-322.

7. On endomorphism rings of primary abelian groups, Math. Ann. 261 (1982), 359-385.

8. Every cotorsion-free algebra is an endomorphism algebra, Math. Z. 181 (1982), 451-470.

9. L. Fuchs, Infinite abelian groups, Vols. I, II, Academic Press, New York, 1970; 1973.

10. R. Göbel, On stout and slender groups, J. Algebra 35 (1975), 39-55.

11. R. Göbel and B. Wald, Wachstumstypen und schlanke Gruppen, Sympos. Math. 23 (1979), 201-239.

12. R. Göbel and S. Shelah, Semi-rigid classes of cotorsion-free abelian groups, J. Algebra (submitted).

13. R. Göbel, Überabzählbare abelsche Gruppen, Westdeutscher Verlag, Oldenburg (to appear).

14. I. Kaplansky, Infinite abelian groups, Univ. of Michigan Press, Ann Arbor, 1971.

15. W. Liebert, Endomorphism rings of abelian p-groups, Studies on Abelian Groups (Symposium, Montpellier, 1967), Springer- Verlag, Berlin, 1968, pp. 239-258. 
16. K. Meinel, Superdecomposable modules over integral domains, Arch. Math. (Basel) 39 (1982), 11-18.

17. A. Orsatti, Anelli dei endomorfismi di gruppi Abeliani senza torsione, Sympos. Math. 8 (1972), 179-191.

18. A class of rings which are the endomorphism rings of some torsion-free abelian groups, Ann. Scuola Norm. Sup. Pisa Cl. Sci. (4) 23 (1969), 143-153.

19. L. Salce and F. Menegazzo, Abelian groups whose endomorphism ring is linearly compact, Rend. Sem. Mat. Univ. Padova 53 (1975), 315-325.

Fachbereich 6, Mathematik, Universität EsSEn, Gesamthochschule D-4300 EsSEN 1, Federal REPUBLIC OF GERMANY 